\title{
A Closer Look at Corrugated Au Tips
}

Ashish Bhattarai, ${ }^{1, \bullet}$ Kevin Crampton, ${ }^{1, \bullet}$ Alan G. Joly, ${ }^{1}$ Chih-Feng Wang, ${ }^{1}$ Zachary D. Schultz, ${ }^{2}$ and Patrick Z. El-Khoury, ${ }^{\text {* }}$

${ }^{1}$ Physical Sciences Division, Pacific Northwest National Laboratory, P.O. Box 999, Richland, WA 99352, USA, ${ }^{2}$ Department of Chemistry and Biochemistry, The Ohio State University, Columbus, $\mathrm{OH} 43210$, USA

-These authors contributed equally to this work

*patrick.elkhoury@pnnl.gov 


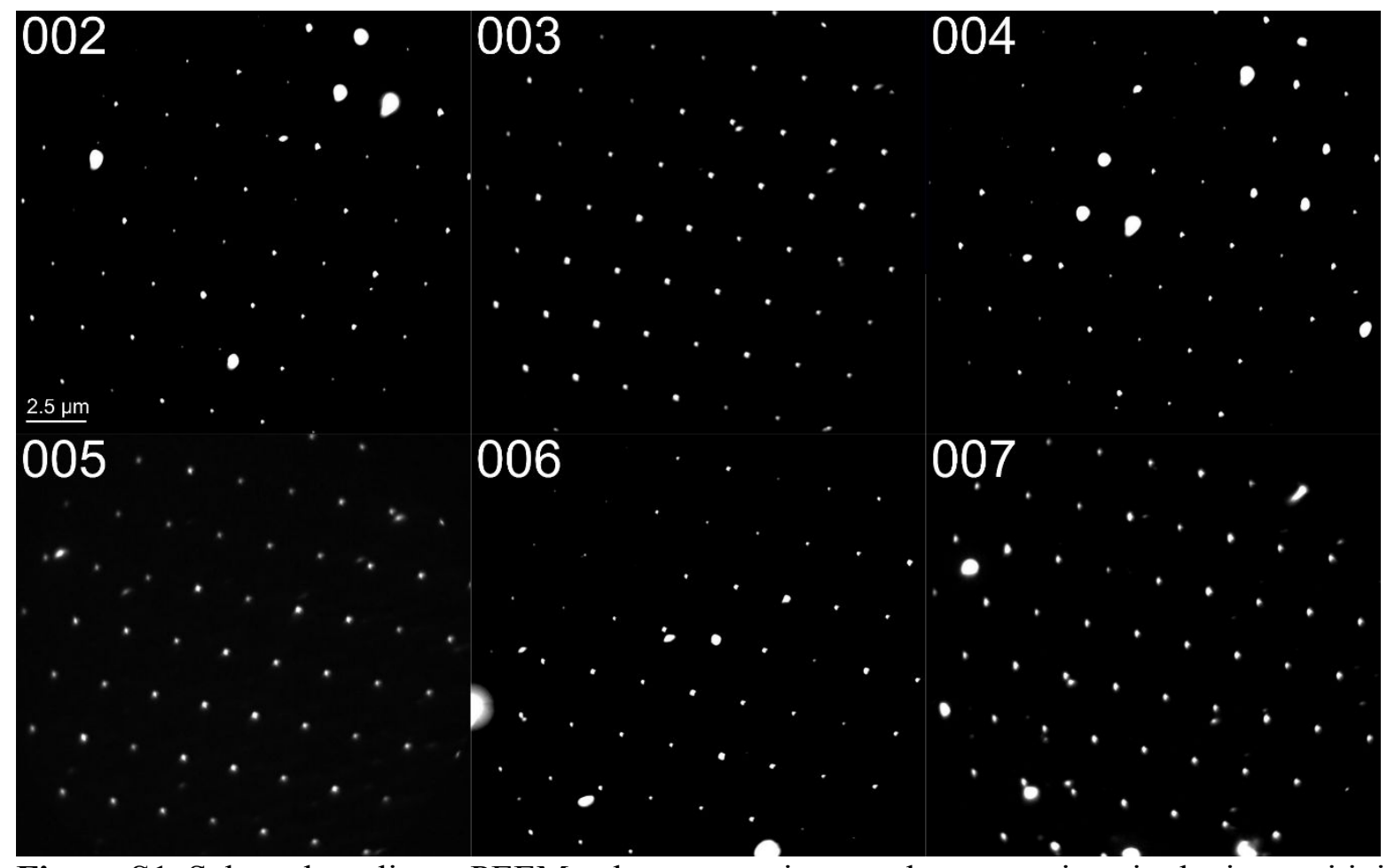

Figure S1. Selected nonlinear PEEM enhancement images demonstrating single tip sensitivity. These images form the basis for the analysis shown in Figure 2 of the main text. 


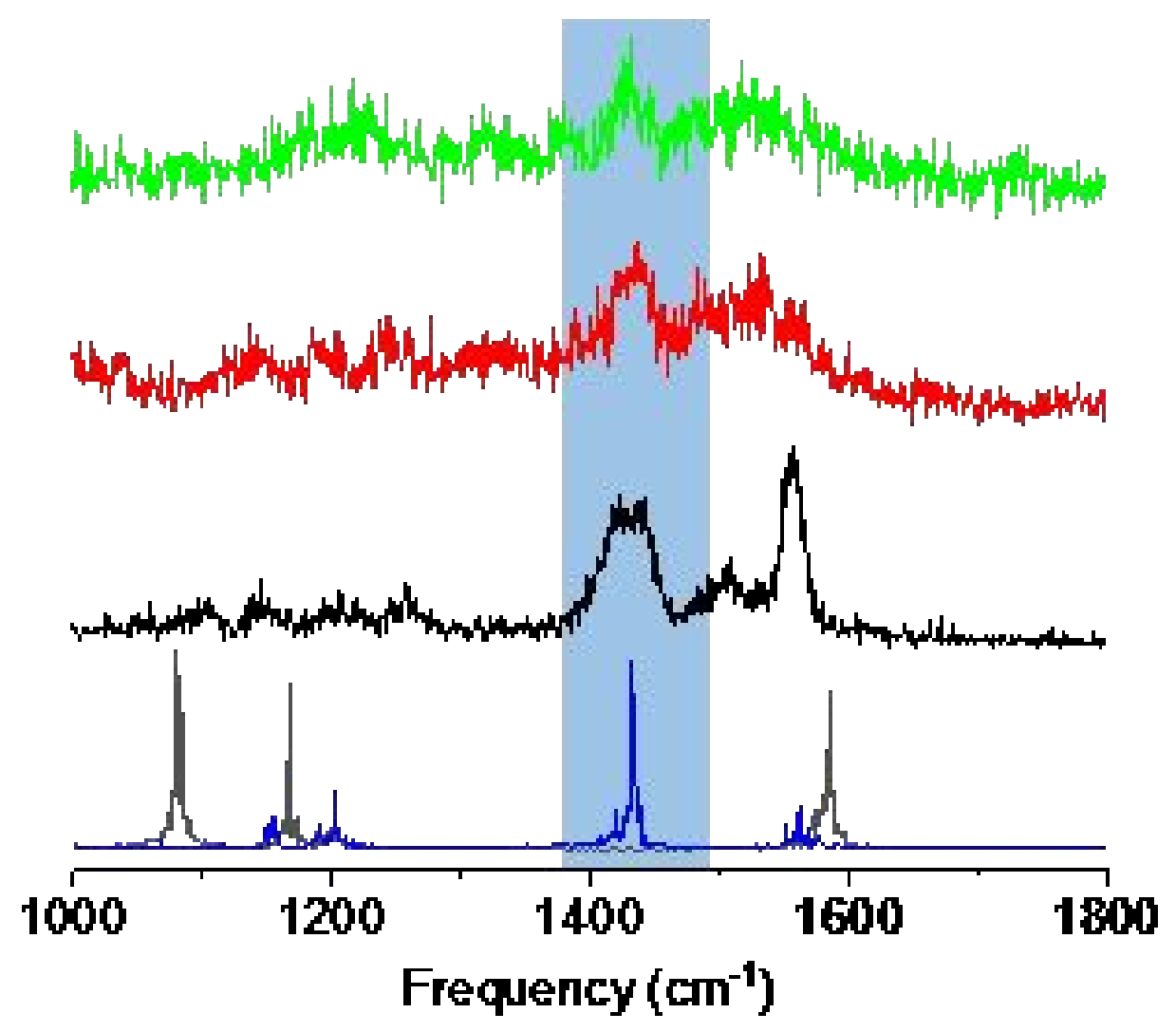

Figure S2. Selected TERS spectra (top three traces) that exhibit a $\sim 1430 \mathrm{~cm}^{-1}$ peak that can be uniquely assigned to anionic TBN. The lowest 2 overlapped spectra in the plot are the theoretical AIMD-Raman spectra of the neutral (black) and anionic (blue) forms of TBN. Both the appearance of the $\sim 1430 \mathrm{~cm}^{-1}$ band and the diming of the lower frequency vibrations of the neutral form (in the 1050-1200 $\mathrm{cm}^{-1}$ spectral region) are indicative anionic TBN species. The same conclusion is reached through a detailed analysis of the nitriles stretching frequencies of the neutral and anionic form in the main text. 


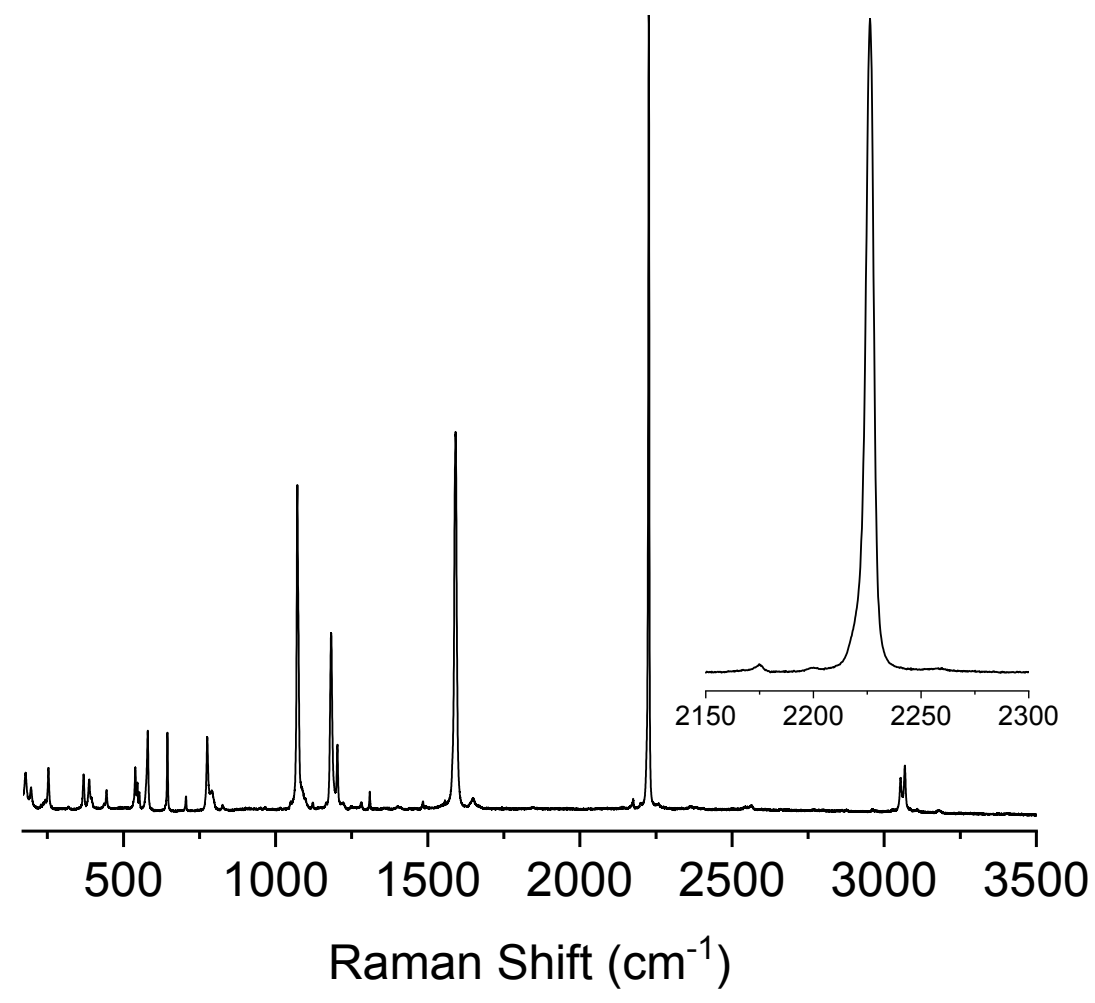

Figure S3. Raman spectrum of TBN (powder, $633 \mathrm{~nm}, 50 \mu \mathrm{w} / \mu \mathrm{m}^{2}$ ). The inset shows the CN stretching vibration, which is centered at $2226.3 \mathrm{~cm}^{-1}$. 